This item was submitted to Loughborough's Research Repository by the author.

Items in Figshare are protected by copyright, with all rights reserved, unless otherwise indicated.

\title{
Extending two fixpoint theorems of Langley and Zheng
}

PLEASE CITE THE PUBLISHED VERSION

LICENCE

CC BY-NC-ND 4.0

\section{REPOSITORY RECORD}

Clifford, E.F.. 2019. "Extending Two Fixpoint Theorems of Langley and Zheng”. figshare. https://hdl.handle.net/2134/374. 


\title{
Extending Two Fixpoint Theorems of Langley and Zheng
}

\author{
E. F. Clifford
}

\begin{abstract}
We extend two theorems on fixpoints of $f(z)$ by Langley and Zheng [1] to the consideration of points where $f(z)=Q(z)$ for some rational function $Q$ such that $Q(\infty)=\infty$. In addition, we extend the class of functions $f$ from transcendental entire functions to meromorphic functions with relatively few poles.
\end{abstract}

Mathematics Subject Classification (1991): 30D30, 30D35

Keywords: value distribution, meromorphic functions, Nevanlinna theory

\section{Introduction}

Let $\mathcal{B}$ denote the class of functions $f$ meromorphic in the plane, for which the set of finite singular values of the inverse function $f^{-1}$ is bounded, that is, the class of all functions $f$ whose set of finite asymptotic and critical values is bounded. This class $\mathcal{B}$ has been considered extensively in iteration theory, see [2], [3], [1].

In [1], Langley and Zheng prove the following two fixpoint theorems, for certain types of functions in the class $\mathcal{B}$.

Theorem 1.1 (Langley and Zheng, [1]) Let $0<\alpha<1$. There is a positive constant c, depending only on $\alpha$, such that if $f$ is a transcendental entire function in the class $\mathcal{B}$, then there are infinitely many fixpoints $z$ satisfying

$$
f(z)=z, \quad\left|f^{\prime}(z)\right|>c \log M(\alpha|z|, f) .
$$

Theorem 1.2 (Langley and Zheng, [1]) Let $f$ be a meromorphic function in the class $\mathcal{B}$, with order $\infty \geq \rho(f)>\mu>0$. Then $f$ has infinitely many fixpoints $z$ with

$$
f(z)=z, \quad\left|f^{\prime}(z)\right|>|z|^{\mu / 2} .
$$

Given a transcendental function $f$ and a rational function $Q$, we define a $Q$-point of $f$ to be any solution $z_{0}$ of the equation $f(z)=Q(z)$. We then extend these two fixpoint theorems to Q-point theorems. In particular, we extend them to the case where $f(z)$ is equal to a rational function $Q(z)$ such that $Q(\infty)=\infty$, that is, where $Q$ is a rational function with a pole of multiplicity $p \geq 1$ at $\infty$.

In addition, we further extend Theorem 1.1 to transcendental meromorphic functions $f$ such that $\delta(\infty, f)>0$, that is, meromorphic functions with relatively few poles. We state the extended theorems as follows. 
Theorem 1.3 Let $0<\alpha<1$. There is a positive constant c, depending only on $\alpha$, such that if $f$ is a transcendental meromorphic function in the class $\mathcal{B}$ with $\delta(\infty, f)>0$, and $Q$ is a rational function with a pole of multiplicity $p \geq 1$ at $\infty$, then there are infinitely many points $z$ satisfying

$$
f(z)=Q(z), \quad\left|f^{\prime}(z)\right|>c \delta(\infty, f) \frac{|Q(z)|}{|z|} T(\alpha|z|, f)
$$

Theorem 1.4 Let $f$ be a meromorphic function in the class $\mathcal{B}$, with order

$\infty \geq \rho(f)>\mu>0$. Let $Q$ be a rational function with a pole of multiplicity $p \geq 1$ at $\infty$. Then $f$ has infinitely many points $z$ with

$$
f(z)=Q(z), \quad\left|f^{\prime}(z)\right|>|z|^{\mu / 2+p-1}
$$

In $\S 2$ we prove a lemma that is used in the proof of Theorem 1.3 and Theorem 1.4 . In $\S 3$ and $\S 4$ we prove Theorem 1.3 and Theorem 1.4 respectively.

\section{A useful lemma}

The following lemma is used in the proof of Theorem 1.3 and Theorem 1.4. We note that it is an extension of [1, Lemma 1], and we will provide the proof for completeness.

Lemma 2.1 Suppose that $f$ is a transcendental meromorphic function in the class $\mathcal{B}$. Let $Q$ be a rational function such that $Q(\infty)=\infty$. Define a function $G$ by

$$
G(z)=f(z) / Q(z), \quad G^{\prime}(z) / G(z)=f^{\prime}(z) / f(z)-Q^{\prime}(z) / Q(z) .
$$

Suppose that $\delta$ is a positive constant. Then there exists a positive constant $\epsilon$ such that the following is true. If $\left|z_{1}\right|$ is large and $\left|G\left(z_{1}\right)-1\right|<\frac{1}{4} \epsilon$, then $z_{1}$ lies in a component $C$ of the set $\left\{z:\left|G(z)-G\left(z_{1}\right)\right|<\frac{1}{2} \epsilon\right\}$, such that $C$ is contained in $B\left(z_{1}, \delta\left|z_{1}\right|\right)$. Define a function $H$ by

$$
H(z)=\frac{2}{\epsilon}\left(G(z)-G\left(z_{1}\right)\right)
$$

Then $C$ is mapped conformally onto $B(0,1)$ by $H$. Furthermore, $\left|Q(z) G^{\prime}(z)\right|$ is large on $C$, and given any $z_{0} \in C$ such that $\left|G\left(z_{0}\right)-G\left(z_{1}\right)\right|<\frac{1}{4} \epsilon$, we have that

$$
\frac{1}{12}\left|G^{\prime}\left(z_{1}\right)\right| \leq\left|G^{\prime}\left(z_{0}\right)\right| \leq \frac{27}{4}\left|G^{\prime}\left(z_{1}\right)\right|
$$

The following two results are used in the proof of Lemma 2.1.

Lemma 2.2 (Eremenko, Lyubich and Bergweiler, [2], [4], [3], [5]) Suppose that $f$ is a transcendental meromorphic function in the class $\mathcal{B}$. Then there are positive constants $R, S$ and $c$ such that

$$
\left|z f^{\prime}(z) / f(z)\right| \geq c \log ^{+}|f(z) / R|
$$

for $|z|>S$. Here, $R$ and $S$ depend on $f$, and $c$ does not.

Theorem 2.3 (Koebe distortion theorem) Let $f$ be an analytic univalent function on B(0,1). Then for $0<r<1$,

$$
\frac{1-r}{(1+r)^{3}}\left|f^{\prime}(0)\right| \leq \max _{|z| \leq r}\left|f^{\prime}(z)\right| \leq \frac{1+r}{(1-r)^{3}}\left|f^{\prime}(0)\right| .
$$


Proof of Lemma 2.1 First we choose a large positive $R_{1}$ and a small positive constant $\epsilon$ such that $\mid G(z)-$ $1 \mid>\epsilon$ on $|z|=R_{1}$. Suppose that $|z|>R_{1}$ and that $|G(z)-1|<\epsilon$. Then we have that $|G(z)|>\frac{1}{2}$, and so by (3), $|f(z)|>\frac{1}{2}|Q(z)|$. Then by Lemma 2.2 , there is a positive constant $R$ such that

$$
\left|z f^{\prime}(z) / f(z)\right| \geq c \log ^{+}|f(z) / R|>c \log |Q(z)|
$$

where $c$ denotes a positive constant which does not depend on $R_{1}$ or $\epsilon$, but not necessarily the same constant at each occurrence.

For such $z$, we have by (3) that

$$
\left|z G^{\prime}(z) / G(z)\right|=\left|z f^{\prime}(z) / f(z)-z Q^{\prime}(z) / Q(z)\right| \geq\left|z f^{\prime}(z) / f(z)\right|-\left|z Q^{\prime}(z) / Q(z)\right| .
$$

We recall that $|G(z)-1|<\epsilon$ and so $|G(z)|$ is close to 1 , and since $Q(\infty)=\infty$ and $z$ is large, we have that $\left|z Q^{\prime}(z) / Q(z)\right|=O(1)$ as $z \rightarrow \infty$. Then by (6) and (7) we have that

$$
\left|z G^{\prime}(z)\right|>R_{2}=c \log \left|Q\left(R_{1}\right)\right|
$$

Then since $Q(\infty)=\infty$ and $z$ is large, we have that $|Q(z)| \geq c|z|$ and so,

$$
\left|Q(z) G^{\prime}(z)\right|>R_{3}=c R_{2} .
$$

Suppose now that $\delta$ is a positive constant and that $z_{1}$ is as in the statement of the lemma, with $\left|z_{1}\right|>2 R_{1}$. That is, $\left|G\left(z_{1}\right)-1\right|<\frac{1}{4} \epsilon$, and $z_{1}$ lies in a component $C$ of the set $\left\{z:\left|G(z)-G\left(z_{1}\right)\right|<\frac{1}{2} \epsilon\right\}$. Let $H$ be defined as in the statement of the lemma, that is, $H(z)=\frac{2}{\epsilon}\left(G(z)-G\left(z_{1}\right)\right)$. Then $H\left(z_{1}\right)=0$ and $\left|H^{\prime}\left(z_{1}\right)\right|=\frac{2}{\epsilon}\left|G^{\prime}\left(z_{1}\right)\right|>2 R_{2} / \epsilon\left|z_{1}\right|$ by (8), and so $H^{\prime}\left(z_{1}\right) \neq 0$. Then $H$ is a conformal mapping in a neighbourhood of $z_{1}$.

Next define

$$
h(w)=\sum_{k=0}^{\infty} c_{k} w^{k}, \quad c_{0}=z_{1},
$$

to be that branch of the inverse function $H^{-1}$ which maps 0 to $z_{1}$, and let $r_{1}$ be the radius of convergence of this series. Then, by a standard compactness argument, there is some $w^{*}$ with $w^{*}=r_{1} e^{i \theta^{*}}$, for some real $\theta^{*}$, such that $h$ has no analytic continuation to a neighbourhood of $w^{*}$. Thus the image of the path $w=t e^{i \theta^{*}}$, $0 \leq t<r_{1}$, under $h$ must, as $t \rightarrow r_{1}$, either tend to $\infty$ or to a multiple point $z^{*}$ of $H$, with $H\left(z^{*}\right)=w^{*}$.

Set $r_{2}=\min \left\{r_{1}, 1\right\}$. Let $\gamma$ be the path $\gamma(t)=t e^{i \theta^{*}}, 0 \leq t<r_{2}$. For $|z|=R_{1}$, we have that

$$
|H(z)|=\frac{2}{\epsilon}\left|G(z)-G\left(z_{1}\right)\right| \geq \frac{2}{\epsilon}\left(|G(z)-1|-\left|G\left(z_{1}\right)-1\right|\right)>\frac{2}{\epsilon}\left(\epsilon-\frac{1}{4} \epsilon\right)=\frac{3}{2},
$$

and since $h(0)=z_{1}$ we must have that the image path $h(\gamma)$ lies in $|z|>R_{1}$. Now $H(h(w))=w$, which by differentiation gives $(H(h(w)))^{\prime}=H^{\prime}(z) h^{\prime}(w)=1$ where $z=h(w)$. Then,

$$
h(w) / h^{\prime}(w)=z H^{\prime}(z)=\frac{2}{\epsilon} z G^{\prime}(z)
$$

and this is large on $\gamma$ by (8). Then for $w$ on $\gamma$ we have by (8) and (11) that

$$
\begin{aligned}
\log \left|h(w) / z_{1}\right| & \leq \int_{0}^{|w|}\left|h^{\prime}\left(s e^{i \theta^{*}}\right) / h\left(s e^{i \theta^{*}}\right)\right| d s \\
& \leq \frac{\epsilon}{2} \frac{|w|}{\inf _{z \in h(\gamma)}\left|z G^{\prime}(z)\right|} \leq \frac{\epsilon}{2 R_{2}} .
\end{aligned}
$$


Then if $\epsilon$ was chosen small enough, the path $h(\gamma)$ lies in $B\left(z_{1}, \delta\left|z_{1}\right|\right)$, and replacing $\theta^{*}$ by any $\theta \in[0,2 \pi]$ we see that $h\left(B\left(0, r_{2}\right)\right) \subseteq B\left(z_{1}, \delta\left|z_{1}\right|\right)$.

On the path $h(\gamma)$ we have

$$
|G(z)-1| \leq\left|G(z)-G\left(z_{1}\right)\right|+\left|G\left(z_{1}\right)-1\right|<\frac{1}{2} \epsilon+\frac{1}{4} \epsilon=\frac{3}{4} \epsilon,
$$

and, using (8), we have that $\left|H^{\prime}(z)\right|=\frac{2}{\epsilon}\left|G^{\prime}(z)\right| \geq 2 R_{2} / \epsilon|z|$. In particular, we have that $h(\gamma)$ is bounded and does not tend to a critical point of $H$. Since $r_{2}=\min \left\{r_{1}, 1\right\}$, we must then have that $r_{1} \geq 1$. Thus, $C$ is contained in $B\left(z_{1}, \delta\left|z_{1}\right|\right)$ and is mapped conformally onto $B(0,1)$ by $H$. Furthermore, by $(9),\left|Q(z) G^{\prime}(z)\right|$ is large on $C$.

Next, let $z_{0} \in C$ such that $\left|G\left(z_{0}\right)-G\left(z_{1}\right)\right|<\frac{1}{4} \epsilon$. Then $\left|H\left(z_{0}\right)\right|=\frac{2}{\epsilon}\left|G\left(z_{0}\right)-G\left(z_{1}\right)\right|<\frac{1}{2}$. Then since $h$ is univalent on $B(0,1)$, there exists some $w_{0} \in B(0,1)$, such that $h\left(w_{0}\right)=z_{0}$. Further, $\left|w_{0}\right|<\frac{1}{2}$, and so by the Koebe distortion theorem we have,

$$
\frac{4}{27}\left|h^{\prime}(0)\right| \leq\left|h^{\prime}\left(w_{0}\right)\right| \leq 12\left|h^{\prime}(0)\right| .
$$

Then since $H^{\prime}(z) h^{\prime}(w)=1$ we have $h^{\prime}(w)=1 / H^{\prime}(z)$ for $w \in B(0,1)$, and in particular, $h^{\prime}(0)=1 / H^{\prime}\left(z_{1}\right)$ and $h^{\prime}\left(w_{0}\right)=1 / H^{\prime}\left(z_{0}\right)$. Also, by $(4), H^{\prime}(z)=\frac{2}{\epsilon} G^{\prime}(z)$, and so by (12) we have

$$
\frac{1}{12}\left|G^{\prime}\left(z_{1}\right)\right| \leq\left|G^{\prime}\left(z_{0}\right)\right| \leq \frac{27}{4}\left|G^{\prime}\left(z_{1}\right)\right| .
$$

\section{Proof of Theorem 1.3}

We need the following theorem.

Theorem 3.1 (Toppila and Winkler, [6]) Let $f$ be a transcendental meromorphic function of order $\lambda$ such that $\delta(\infty, f)>0$. Then

$$
\limsup _{z \rightarrow \infty, z \in E(f)} \frac{|z| f^{\sharp}(z)}{T(|z|, f)} \geq A_{0} \delta(\infty, f)(1+\lambda)
$$

where $E(f)=\{z:|f(z)|=1\}, A_{0}$ is a positive absolute constant and $f^{\sharp}(z)=\frac{\left|f^{\prime}(z)\right|}{1+|f(z)|^{2}}$ is the spherical derivative.

We now prove Theorem 1.3.

Proof of Theorem 1.3 Let $0<\alpha<1$ and let $R$ be a large positive constant. Let $\delta$ and $\epsilon$ be as in Lemma 2.1 , with $\frac{1}{1+\delta}>\alpha$. Define functions $G$ and $f_{1}$ by

$$
G(z)=\frac{f(z)}{Q(z)}=1+\frac{\epsilon f_{1}(z)}{16} .
$$

Then $f_{1}(z)=\frac{16}{\epsilon}\left(\frac{f(z)}{Q(z)}-1\right)=c_{1} \frac{f(z)}{Q(z)}-c_{2}$ where $c_{j}$ denotes a positive constant not depending on $R$. Then since $f$ is a transcendental meromorphic function and since $Q$ is a rational function, we have that $f_{1}$ is a transcendental meromorphic function. 
Further, we have that $\delta\left(\infty, f_{1}\right)>0$ since $f(z)=\left(c_{3} f_{1}(z)+c_{4}\right) Q(z)$ and,

$$
\begin{aligned}
0<\delta(\infty, f) & =\delta\left(\infty,\left(c_{3} f_{1}+c_{4}\right) Q\right) \\
& =\liminf _{r \rightarrow \infty} \frac{m\left(r,\left(c_{3} f_{1}+c_{4}\right) Q\right)}{T\left(r,\left(c_{3} f_{1}+c_{4}\right) Q\right)} \\
& \leq \liminf _{r \rightarrow \infty} \frac{m\left(r, c_{3} f_{1}+c_{4}\right)+m(r, Q)+O(1)}{T\left(r, c_{3} f_{1}+c_{4}\right)-T(r, Q)-O(1)} \\
& =\liminf _{r \rightarrow \infty} \frac{m\left(r, f_{1}\right)+O(\log r)+O(1)}{T\left(r, f_{1}\right)-O(\log r)-O(1)} \\
& =\delta\left(\infty, f_{1}\right),
\end{aligned}
$$

since $Q$ is a rational function and $f_{1}$ is a transcendental function function.

Let $\lambda$ be the order of $f_{1}$, and let $E\left(f_{1}\right)=\left\{z:\left|f_{1}(z)\right|=1\right\}$. Then by Theorem 3.1, we have that

$$
\limsup _{z \rightarrow \infty, z \in E\left(f_{1}\right)} \frac{|z| f_{1}^{\sharp}(z)}{T\left(|z|, f_{1}\right)} \geq A_{0} \delta\left(\infty, f_{1}\right)(1+\lambda),
$$

where $A_{0}$ is a positive absolute constant. We note that for $z \in E\left(f_{1}\right)$ we have that $f_{1}^{\sharp}(z)=\frac{\left|f_{1}^{\prime}(z)\right|}{1+\left|f_{1}(z)\right|^{2}}=\frac{\left|f_{1}^{\prime}(z)\right|}{2}$. Then we have

$$
\limsup _{z \rightarrow \infty, z \in E\left(f_{1}\right)} \frac{|z|\left|f_{1}^{\prime}(z)\right|}{T\left(|z|, f_{1}\right)}>d_{1}=A_{0} \delta\left(\infty, f_{1}\right),
$$

and so there exists a sequence $\left(\zeta_{n}\right)$ in $E\left(f_{1}\right), \zeta_{n} \rightarrow \infty$ as $n \rightarrow \infty$, such that

$$
\frac{\left|\zeta_{n}\right|\left|f_{1}^{\prime}\left(\zeta_{n}\right)\right|}{T\left(\left|\zeta_{n}\right|, f_{1}\right)}>d_{1}
$$

Thus we can choose $z_{1}$ in $E\left(f_{1}\right)$ arbitrarily large, and in particular such that $\left|z_{1}\right|>R$, with

$$
\left|z_{1}\right|\left|f_{1}^{\prime}\left(z_{1}\right)\right|>d_{1} T\left(\left|z_{1}\right|, f_{1}\right) .
$$

Next, since $f(z)=\left(c_{3} f_{1}(z)+c_{4}\right) Q(z)$ we have that

$$
\begin{aligned}
T\left(\left|z_{1}\right|, f\right) & =T\left(\left|z_{1}\right|,\left(c_{3} f_{1}+c_{4}\right) Q\right) \\
& \leq T\left(\left|z_{1}\right|, c_{3} f_{1}+c_{4}\right)+T\left(\left|z_{1}\right|, Q\right) \\
& \leq T\left(\left|z_{1}\right|, f_{1}\right)+O\left(\log \left|z_{1}\right|\right),
\end{aligned}
$$

and so, if $R$ is large enough,

$$
T\left(\left|z_{1}\right|, f_{1}\right) \geq T\left(\left|z_{1}\right|, f\right)-O\left(\log \left|z_{1}\right|\right)>\frac{1}{2} T\left(\left|z_{1}\right|, f\right),
$$

since $f$ is a transcendental function. Then since $\left|G^{\prime}(z)\right|=\frac{\epsilon}{16}\left|f_{1}^{\prime}(z)\right|$, we have by (14) and (15) that

$$
\left|G^{\prime}\left(z_{1}\right)\right|>\frac{\epsilon}{16} \frac{d_{1}}{\left|z_{1}\right|} T\left(\left|z_{1}\right|, f_{1}\right)>\frac{d_{2}}{\left|z_{1}\right|} T\left(\left|z_{1}\right|, f\right),
$$

where $d_{2}=\frac{\epsilon}{32} d_{1}$.

We recall that $G(z)=1+\frac{\epsilon f_{1}(z)}{16}$, and thus since $z_{1} \in E\left(f_{1}\right)$, we have that $\left|G\left(z_{1}\right)-1\right|=\frac{\epsilon}{16}<\frac{\epsilon}{4}$. Then by Lemma 2.1, $z_{1}$ lies in a component $C$ of the set $\left\{z:\left|G(z)-G\left(z_{1}\right)\right|<\frac{1}{2} \epsilon\right\}$ such that $C$ is contained in $B\left(z_{1}, \delta\left|z_{1}\right|\right)$. Also, by Lemma $2.1, C$ is mapped conformally onto $B(0,1)$ by the function $H(z)=\frac{2}{\epsilon}(G(z)-$ 
$\left.G\left(z_{1}\right)\right)$. Then we can choose a point $z_{2}$ in $C$ such that $G\left(z_{2}\right)=1$ and $\left|G\left(z_{2}\right)-G\left(z_{1}\right)\right|<\frac{1}{4} \epsilon$. Furthermore, we have by (5) that,

$$
\frac{1}{12}\left|G^{\prime}\left(z_{1}\right)\right| \leq\left|G^{\prime}\left(z_{2}\right)\right| \leq \frac{27}{4}\left|G^{\prime}\left(z_{1}\right)\right|
$$

and so by (16) we have

$$
\left|G^{\prime}\left(z_{2}\right)\right|>\frac{d_{3}}{\left|z_{1}\right|} T\left(\left|z_{1}\right|, f\right)
$$

where $d_{3}=\frac{d_{2}}{12}$ Then, since we have by (3) that,

$$
z_{2} \frac{f^{\prime}\left(z_{2}\right)}{f\left(z_{2}\right)}=z_{2} \frac{G^{\prime}\left(z_{2}\right)}{G\left(z_{2}\right)}+z_{2} \frac{Q^{\prime}\left(z_{2}\right)}{Q\left(z_{2}\right)}
$$

and since $f\left(z_{2}\right)=Q\left(z_{2}\right)$ and $G\left(z_{2}\right)=1$, we have that

$$
\left|z_{2} \frac{f^{\prime}\left(z_{2}\right)}{Q\left(z_{2}\right)}\right| \geq\left|z_{2} G^{\prime}\left(z_{2}\right)\right|-\left|z_{2} \frac{Q^{\prime}\left(z_{2}\right)}{Q\left(z_{2}\right)}\right| .
$$

Now since $Q$ is a rational function with a pole of multiplicity $p \geq 1$ at $\infty$, we have that $\left|z \frac{Q^{\prime}(z)}{Q(z)}\right|=O(1)$ as $z \rightarrow \infty$. Since $z_{2} \in B\left(z_{1}, \delta\left|z_{1}\right|\right)$, we have that $\left|z_{1}\right| \leq\left|z_{1}-z_{2}\right|+\left|z_{2}\right| \leq \delta\left|z_{1}\right|+\left|z_{2}\right|$, which gives that $\left|\frac{z_{2}}{z_{1}}\right| \geq 1-\delta$. Then by (17) and (18), we have that

$$
\begin{aligned}
\left|z_{2} \frac{f^{\prime}\left(z_{2}\right)}{Q\left(z_{2}\right)}\right| & \geq\left|z_{2} G^{\prime}\left(z_{2}\right)\right|-O(1) \\
& \geq \frac{1}{2}\left|\frac{z_{2}}{z_{1}}\right| d_{3} T\left(\left|z_{1}\right|, f\right) \\
& \geq \frac{1-\delta}{2} d_{3} T\left(\left|z_{1}\right|, f\right) \\
& \geq \frac{1-\delta}{2} d_{3} T\left(\alpha\left|z_{2}\right|, f\right) .
\end{aligned}
$$

Then since $d_{3}=\frac{\epsilon}{384} A_{0} \delta(\infty, f)$ and $A_{0}$ is an absolute constant, we have that

$$
\left|f^{\prime}\left(z_{2}\right)\right|>c \delta(\infty, f) \frac{\left|Q\left(z_{2}\right)\right|}{\left|z_{2}\right|} T\left(\alpha\left|z_{2}\right|, f\right) .
$$

where $c$ is a positive constant depending only on $\alpha$.

\section{Proof of Theorem 1.4}

We need the following theorem.

Theorem 4.1 (Langley and Zheng, [1]) Let $f$ be a transcendental meromorphic function in the class $\mathcal{B}$. If $Q$ is a rational function with a pole of multiplicity $p \geq 1$ at $\infty$, then

$$
m\left(r, \frac{1}{f-Q}\right)=O(\log r T(r, f))
$$

as $r \rightarrow \infty$ outside a set of finite measure.

We note that the Nevanlinna counting function $N\left(r, \frac{1}{f-z}\right)$ counts the poles of $\frac{1}{f-z}$, that is, the fixpoints of $f$. In [1] the following implication of Theorem 4.1 is noted, namely, that $N\left(r, \frac{1}{f-z}\right)$ cannot satisfy $N\left(r, \frac{1}{f-z}\right)=$ $o(T(r, f))$ as $r \rightarrow \infty$. We extend this to where $Q$ is a rational function such that $Q(\infty)=\infty$, and state the result as a corollary. We provide a proof for completeness. 
Corollary 4.2 Let $f$ and $Q$ be as in the statement of Theorem 1.4. Then the Nevanlinna counting function $N\left(r, \frac{1}{f-Q}\right)$, of points where $f(z)=Q(z)$, cannot satisfy $N\left(r, \frac{1}{f-Q}\right)=o(T(r, f))$ as $r \rightarrow \infty$. Also, for $\sigma>0$, we may choose arbitrarily large $r$ such that there are at least $2 r^{\sigma}$ Q-points $z_{j}$, such that $f\left(z_{j}\right)=Q\left(z_{j}\right)$, in the annulus $\frac{1}{2} r \leq|z| \leq r$.

Proof Since $Q$ is a rational function we have that

$$
T(r, f-Q) \leq T(r, f)+T(r, Q)+O(1)=T(r, f)+O(\log r)+O(1)
$$

and that,

$$
T(r, f)=T(r, f-Q+Q) \leq T(r, f-Q)+T(r, Q)+O(1)=T(r, f-Q)+O(\log r)+O(1) .
$$

Thus

$$
|T(r, f)-T(r, f-Q)| \leq O(\log r)+O(1)
$$

and since, $T\left(r, \frac{1}{f-Q}\right)=T(r, f-Q)+O(1)$ we have that

$$
\left|T(r, f)-T\left(r, \frac{1}{f-Q}\right)-O(1)\right| \leq O(\log r)+O(1) .
$$

Then since $T\left(r, \frac{1}{f-Q}\right)=N\left(r, \frac{1}{f-Q}\right)+m\left(r, \frac{1}{f-Q}\right)$ we have that

$$
\left|T(r, f)-T\left(r, \frac{1}{f-Q}\right)-O(1)\right| \geq\left|T(r, f)-N\left(r, \frac{1}{f-Q}\right)\right|-\left|m\left(r, \frac{1}{f-Q}\right)+O(1)\right| .
$$

We may assume by (19) that $m\left(r, \frac{1}{f-Q}\right)=O(\log r T(r, f))$ as $r \rightarrow \infty$, with $r \notin E$, for some set $E$ of finite measure. Then by (21) and Theorem 4.1 we have

$$
\begin{aligned}
\left|T(r, f)-N\left(r, \frac{1}{f-Q}\right)\right| & \leq O(\log r)+O(1)+\left|m\left(r, \frac{1}{f-Q}\right)+O(1)\right| \\
& =O(\log r)+O(1)+O(\log r T(r, f)), \quad r \notin E .
\end{aligned}
$$

Then since $f$ is a transcendental function, we have that $N\left(r, \frac{1}{f-Q}\right)$ cannot satisfy $N\left(r, \frac{1}{f-Q}\right)=o(T(r, f))$ as $r \rightarrow \infty$.

Next we show that all large $z_{j}$ such that $f\left(z_{j}\right)=Q\left(z_{j}\right)$ are simple zeros of $f-Q$. Let $z_{0}$ be large and suppose $f\left(z_{0}\right)=Q\left(z_{0}\right)$. Then since $f$ and $Q$ satisfy the hypotheses of Lemma 2.1 and Lemma 2.2 , we have by (6) that

$$
\left|z_{0} f^{\prime}\left(z_{0}\right) / f\left(z_{0}\right)\right|>R_{1}=c \log \left|Q\left(z_{0}\right)\right|
$$

where $c$ is a positive constant. Also, since $Q$ is a rational function with a pole of multiplicity $p \geq 1$ at $\infty$, we have that $\left|\frac{Q^{\prime}\left(z_{0}\right)}{Q\left(z_{0}\right)}\right| \leq \frac{p}{\left|z_{0}\right|}(1+o(1))$. Then, by $(22)$ and since $f\left(z_{0}\right)=Q\left(z_{0}\right)$, we have that

$$
\left|f^{\prime}\left(z_{0}\right)\right|>\frac{R_{1}}{\left|z_{0}\right|}\left|Q\left(z_{0}\right)\right| \geq \frac{p}{\left|z_{0}\right|}(1+o(1))\left|Q\left(z_{0}\right)\right| \geq\left|Q^{\prime}\left(z_{0}\right)\right|
$$

since we can choose $z_{0}$ so large that $p / R_{1}$ is very small. Then this gives that $f^{\prime}\left(z_{0}\right)-Q^{\prime}\left(z_{0}\right) \neq 0$ and so $z_{0}$ is a simple zero of $f-Q$.

Suppose now that $r_{0} \geq 0$ is such that for $r \geq r_{0}$, there are less than $2 r^{\sigma}$ points $z_{j}$, such that $f\left(z_{j}\right)=Q\left(z_{j}\right)$, in $\frac{r}{2} \leq|z| \leq r$. Then for $m \in \mathbb{N}$ we have that

$$
n\left(2^{m} r_{0}, \frac{1}{f-Q}\right)<n\left(r_{0}, \frac{1}{f-Q}\right)+2\left[\left(2^{m} r_{0}\right)^{\sigma}+\left(2^{m-1} r_{0}\right)^{\sigma}+\ldots+\left(2 r_{0}\right)^{\sigma}\right] .
$$


Let $r$ be large, with, in particular, $r>r_{0}$. Then there exists $m \in \mathbb{N}$ such that $2^{m-1} r_{0} \leq r<2^{m} r_{0}$. Then by (23) we have

$$
\begin{aligned}
n\left(r, \frac{1}{f-Q}\right) & \leq n\left(2^{m} r_{0}, \frac{1}{f-Q}\right) \\
& <n\left(r_{0}, \frac{1}{f-Q}\right)+2\left[\left(2^{m} r_{0}\right)^{\sigma}+\left(2^{m-1} r_{0}\right)^{\sigma}+\ldots+\left(2 r_{0}\right)^{\sigma}\right] \\
& =c_{1}+2 r_{0}^{\sigma}\left[\frac{2^{\sigma}\left(2^{\sigma m}-1\right)}{2^{\sigma}-1}\right] \\
& \leq c_{1}+c_{2} r^{\sigma}
\end{aligned}
$$

where $c_{1}=n\left(r_{0}, \frac{1}{f-Q}\right)$ and $c_{2}$ is a positive constant. Then we have $N\left(r, \frac{1}{f-Q}\right)=O\left(r^{\sigma}\right)$.

Next, by (19), we may choose $r \leq r_{1} \leq 2 r$ such that $m\left(r_{1}, \frac{1}{f-Q}\right)=O\left(\log r_{1} T\left(r_{1}, f\right)\right)$. Then

$$
\begin{aligned}
T\left(r_{1}, \frac{1}{f-Q}\right) & =N\left(r_{1}, \frac{1}{f-Q}\right)+m\left(r_{1}, \frac{1}{f-Q}\right) \\
& =O\left(r_{1}^{\sigma}\right)+O\left(\log r_{1} T\left(r_{1}, f\right)\right),
\end{aligned}
$$

and so,

$$
T\left(r, \frac{1}{f-Q}\right) \leq T\left(r_{1}, \frac{1}{f-Q}\right)=O\left(r^{\sigma}\right) .
$$

Therefore we have that $T(r, f)=O\left(r^{\sigma}\right)$, and thus,

$$
\rho(f)=\limsup _{r \rightarrow \infty} \frac{\log ^{+} T(r, f)}{\log r} \leq \sigma .
$$

This is a contradiction since $\rho(f)>\sigma$. Therefore, we may choose arbitrarily large $r$ such that there are at least $2 r^{\sigma}$ points $z_{j}$ such that $f\left(z_{j}\right)=Q\left(z_{j}\right)$ in the annulus $\frac{1}{2} r \leq|z| \leq r$.

We now prove Theorem 1.4.

Proof of Theorem 1.4 Let $f$ and $Q$ be as in the statement of Theorem 1.4. Choose $\sigma$ with $\rho(f)>\sigma>\mu$. Since $\rho(f)>0$, we have that $f$ is a transcendental function, and so by Corollary 4.2, we can choose arbitrarily large $r$ such that $f$ has at least $2 r^{\sigma}$ points $z_{j}$ such that $f\left(z_{j}\right)=Q\left(z_{j}\right)$ in the annulus $\frac{1}{2} r \leq|z| \leq r$.

Suppose $\delta$ is a small positive constant. Let $G(z)=f(z) / Q(z)$ be as defined in Lemma 2.1. Then for each $z_{j}$ we have that $G\left(z_{j}\right)=1$. Then by Lemma 2.1, we have that to each $z_{j}$ there corresponds a component $C_{j}$ of the set $\left\{z:|G(z)-1|<\frac{1}{2} \epsilon\right\}$, and that each $C_{j}$ is contained in $B\left(z_{j}, \delta\left|z_{j}\right|\right)$. Then choosing $\delta$ small enough gives that each $C_{j}$ lies in the annulus $\frac{1}{4} r \leq|z| \leq 2 r$. These $C_{j}$ are disjoint simple islands since, by Lemma 2.1 , they are mapped conformally onto $B(0,1)$ by the function $H(z)=\frac{2}{\epsilon}\left(G(z)-G\left(z_{j}\right)\right)$.

Let $h_{j}: B(0,1) \rightarrow C_{j}$ be the inverse function of $H$. Then $h_{j}$ is a univalent function on $\mathrm{B}(0,1)$ and by the Koebe distortion theorem we have that for $0<r_{0}<1$,

$$
c_{1}\left|h_{j}^{\prime}(0)\right| \leq \max _{|w| \leq r_{0}}\left|h_{j}^{\prime}(w)\right| \leq c_{2}\left|h_{j}^{\prime}(0)\right|
$$

where $c_{1}$ and $c_{2}$ are positive constants, depending only on $r_{0}$.

In particular, choose $r_{0}=\frac{1}{2}$ and let $\hat{C}_{j}=h_{j}\left(B\left(0, \frac{1}{2}\right)\right)$. Then $\left|h_{j}^{\prime}(w)\right| \geq c_{1}\left|h_{j}^{\prime}(0)\right|$ for $|w| \leq \frac{1}{2}$. Then since $h_{j}^{\prime}(w)=1 / H^{\prime}(z)$ where $z=h_{j}(w)$, and in particular since $h_{j}^{\prime}(0)=1 / H^{\prime}\left(z_{j}\right)=\epsilon / 2 G^{\prime}\left(z_{j}\right)$ we have that

$$
\left|h_{j}^{\prime}(w)\right| \geq c /\left|G^{\prime}\left(z_{j}\right)\right|, \quad \text { for }|w| \leq \frac{1}{2},
$$


where, from here on, $c$ denotes a positive constant, not necessarily the same at each occurrence, but not depending on $r$ or $\sigma$.

Next, since the area of the annulus $\frac{1}{4} r \leq|z| \leq 2 r$ is $c r^{2}$, and since there are at least $2 r^{\sigma}$ disjoint components $\hat{C}_{j}$ in the annulus, we have that at least $r^{\sigma}$ of these $\hat{C}_{j}$ have area at most $c r^{2-\sigma}$. Then, for these $z_{j}$, we have

$$
z_{j} \frac{f^{\prime}\left(z_{j}\right)}{f\left(z_{j}\right)}=z_{j} \frac{G^{\prime}\left(z_{j}\right)}{G\left(z_{j}\right)}+z_{j} \frac{Q^{\prime}\left(z_{j}\right)}{Q\left(z_{j}\right)},
$$

and since $f\left(z_{j}\right)=Q\left(z_{j}\right)$ and $G\left(z_{j}\right)=1$, we have that

$$
\left|z_{j} \frac{f^{\prime}\left(z_{j}\right)}{Q\left(z_{j}\right)}\right| \geq\left|z_{j} G^{\prime}\left(z_{j}\right)\right|-\left|z_{j} \frac{Q^{\prime}\left(z_{j}\right)}{Q\left(z_{j}\right)}\right| .
$$

Now since $Q$ is a rational function with a pole of multiplicity $p \geq 1$ at $\infty$, we have that $\left|z \frac{Q^{\prime}(z)}{Q(z)}\right|=O(1)$ as $z \rightarrow \infty$. Therefore, (25) gives that

$$
\left|z_{j} \frac{f^{\prime}\left(z_{j}\right)}{Q\left(z_{j}\right)}\right| \geq\left|z_{j} G^{\prime}\left(z_{j}\right)\right|-O(1)
$$

Now by $[7$, p.4] and $(24)$ we have that

$$
\text { area of } \hat{C}_{j}=\iint_{B\left(0, \frac{1}{2}\right)}\left|h^{\prime}(w)\right|^{2} d u d v \geq c /\left|G^{\prime}\left(z_{j}\right)\right|^{2} .
$$

And so, since the area of $\hat{C}_{j}$ is at most $c r^{2-\sigma}$ we have that $1 /\left|G^{\prime}\left(z_{j}\right)\right|^{2} \leq c r^{2-\sigma}$, which gives $\left|G^{\prime}\left(z_{j}\right)\right| \geq c r^{\sigma / 2-1}$. Then by (26) and since $r \geq\left|z_{j}\right|$, and $z_{j}$ is large, and $p \geq 1$, we have

$$
\left|z_{j} \frac{f^{\prime}\left(z_{j}\right)}{Q\left(z_{j}\right)}\right| \geq c r^{\sigma / 2}-O(1) \geq c\left|z_{j}\right|^{\sigma / 2}
$$

Then since $\mid Q\left(\left.z_{j}|>c| z_{j}\right|^{p}\right.$, we have that

$$
\left|f^{\prime}\left(z_{j}\right)\right|>c\left|z_{j}\right|^{\sigma / 2+p-1} \geq\left|z_{j}\right|^{\mu / 2+p-1} .
$$

Acknowledgements: This research has been done as part of a $\mathrm{PhD}$ thesis at the University of Nottingham. The author gratefully acknowledges the advice and support of Prof J. K. Langley who suggested these results and provided useful ideas and comments. The author also acknowledges the referee(s) for their valuable contributions to this paper.

\section{References}

[1] J. K. Langley and J. H. Zheng. On the fixpoints, multipliers and value distribution of certain classes of meromorphic functions. Ann. Acad. Sci. Fenn. Math., 23(1):133-150, 1998.

[2] W. Bergweiler. On the zeros of certain homogeneous differential polynomials. Arch. Math. (Basel), 64(3):199-202, 1995.

[3] A. È. Erëmenko and M. Yu. Lyubich. Dynamical properties of some classes of entire functions. Ann. Inst. Fourier (Grenoble), 42(4):989-1020, 1992.

[4] Walter Bergweiler. Iteration of meromorphic functions. Bull. Amer. Math. Soc. (N.S.), 29(2):151-188, 1993. 
[5] P. Rippon and G. Stallard. Iteration of a class of hyperbolic meromorphic functions. Proc. Amer. Math. Soc., 127:3251-3258, 1999.

[6] Sakari Toppila and Jörg Winkler. Some lower bounds for the maximal growth of the spherical derivative. Complex Variables Theory Appl., 2(1):1-25, 1983.

[7] Ch. Pommerenke. Boundary behaviour of conformal maps, volume 299 of Grundlehren der Mathematischen Wissenschaften [Fundamental Principles of Mathematical Sciences]. Springer-Verlag, Berlin, 1992.

Address: Department of Mathematical Sciences, Loughborough University, Leicestershire, LE11 3TU, UK

Email: E.Clifford@lboro.ac.uk

Eingegangen am 12. November 2004 